\title{
Dioniso in Sicilia. Mythos, Symposion, Hades,
} Theatron, Mysteria

("Mesogheia. Studi di storia e archeologia della Sicilia Antica"),

Caltanissetta, Edizioni Lussografica, 2018, pp. 269,

ISBN 978-88-8243-452-6, € 22,00

Nicola Cusumano

\section{(2) OpenEdition}

\section{Edizione digitale}

URL: http://journals.openedition.org/mythos/375

DOI: $10.4000 /$ mythos. 375

ISSN: 2037-7746

\section{Editore}

Salvatore Sciascia Editore

\section{Edizione cartacea}

Data di pubblicazione: 1 dicembre 2018

Paginazione: 159-161

ISBN: 978-88-8241-501-3

ISSN: $1972-2516$

Notizia bibliografica digitale

Nicola Cusumano, « Dioniso in Sicilia. Mythos, Symposion, Hades, Theatron, Mysteria », Mythos [Online], 12 | 2018, online dal 24 septembre 2019, consultato il 25 septembre 2020. URL : http:// journals.openedition.org/mythos/375; DOI : https://doi.org/10.4000/mythos.375 


\section{Simona Modeo}

Dioniso in Sicilia. Mythos, Symposion, Hades, Theatron, Mysteria

("Mesogheia. Studi di storia e archeologia della Sicilia Antica”), Caltanissetta, Edizioni Lussografica, 2018, pp. 269, ISBN 978-88-8243-452-6, € 22,00.

\section{Nicola Cusumano - Università di Palermo - nicola.cusumano25@unipa.it}

Prendendo opportunamente avvio dall'innovativo e per molti versi ancora "fresco" volume di Emanuele Ciaceri (Culti e miti nella storia dell'antica Sicilia, 1911), Simona Modeo sottolinea che il gap documentario lamentato un tempo da Ciaceri a proposito del culto di Dioniso può essere considerato oggi almeno in parte superato grazie all'intensa attività di ricerca dell'ultimo secolo, che apre nuovi orizzonti interpretativi, soprattutto per quanto riguarda l'area anellenica, tanto indigena che fenicio-punica. Partendo da questo assunto S. Modeo esplora le motivazioni di questo culto identificandole in primo luogo nel profilo soterico di Dioniso, in grado di giustificare un ruolo di connettore anche interculturale. L'indagine è distribuita in cinque sezioni che prendono in esame aspetti diversi della divinità e del suo culto. La prima sezione (Mythos. La rappresentazione del mondo dionisiaco, 17-55), a sua volta articolata in tre capitoli, prende in esame la natura polimorfica della divinità, così come è testimoniata ad esempio nell'iconografia vascolare attica, importata in Sicilia dal VI secolo: le formule iconiche sono legate alla c.d. "faccialità" del dio, secondo la formula proposta da J.-P. Vernant. Tanto la committenza greca quanto quelle indigena nell'isola prediligono in questo periodo l'immagine tradizionale della divinità, "paterna e regale", con alcune eccezioni come il Dioniso fanciullo su un cratere proveniente da Sabucina (22). Sembra assente in ambito siceliota la dimensione poliade del dio. Risultano invece presenti nella ceramica attica importata in Sicilia le figure del thiasos: le danze menadiche decorano le ceramiche a figure rosse dal $V$ secolo; in particolare, la presenza di phialai piene di uova testimoniano il rapporto tra dionisismo e orfismo, attestato sia in Sicilia che in Magna Grecia (30). Ugualmente ricco di attestazioni è il tema figurativo delle piante e degli animali legati al dio.

La seconda sezione (Symposion. Dioniso, il vino e il komos, 57-79) si lega all'importante sfera economica della produzione di vino e del suo consumo in Sicilia, con i connessi aspetti rituali e sociali. In una rapida sintesi sulla questione, l'Autrice mette a frutto con perizia gli ampi risultati che sono stati ottenuti dalle ricerche recenti estese a tutto il territorio siciliano, pur concludendo con la dovuta prudenza che non si è ancora giunti ad una situazione documentaria chiaramente traducibile in discorso storico. Gli aspetti simposiali sono tuttavia ben documenti dall'iconografia vascolare: essi gettano luce, grazie soprattutto ai corredi tombali, tanto sugli ambienti sicelioti che su quelli "indigeni". Resta da valutare attraverso quali codici di senso la pratica del simposio, così centrale nella madrepatria, si sia diffusa nelle colonie e poi sia stata 'ritradotta' anche in ambienti anellenici. La terza sezione (Hades. Dioniso e l'al di là, 81-105) affronta la diffusione dell'aspetto funerario di Dioniso nell'isola, a partire dalla relazione vino/morte attestata a più livelli e sorretta da valori escatologici. I dati provenienti dalle necropoli ad incinerazione secondaria in cratere e dall'iconografia vascolare evidenziano una connessione tra Hades e Dioniso che, diversamente da quel che si riscontra nell'ambiente greco peninsulare, qui in Sicilia e in Magna Grecia mostra un valore positivo dando luogo ad una interscambiabilità tra le due divinità. Particolarmente interessante è la prassi dell'incinerazione in cratere che S. Modeo inquadra nel mito del trattamento delle ossa di Achille e nell'omologia vino/fuoco, acutamente studiata da F. Lissarrague: la pratica 
rituale dell'incinerazione nel cratere risulta così collegata ad un processo di trasformazione del corpo omologo alla fermentazione del vino, e in certa misura ad un processo di immersione nel vino che blocca il miasma prodotto dalla morte. II collegamento Dioniso/Hades, sia su ceramica attica che siceliota, emerge anche sul piano iconografico tra la fine del $\mathrm{VI}$ e il V secolo. Altro aspetto di questo legame è suggerito dalla ricca coroplastica liparese a tema "teatrale" che, come ha ben visto nei suoi studi Bernabò Brea, risulta "strettamente connessa al mondo dell'oltretomba” (97). Si aggiunga che la presenza di maschere nelle tombe di Lipari fa pensare ad una possibile offerta a Dioniso in vista dello status post mortem.

Appartenga o no al mito il viaggio di Arione in Italia e in Sicilia, è ormai opinione accolta che forme di spettacolo si siano sviluppate in Italia meridionale fin dall'età arcaica, una tradizione che si collega alle rappresentazioni teatrali siciliane nel $\mathrm{V}$ secolo. Oltre alle fonti letterarie, le testimonianze archeologiche forniscono una gran massa di dati a conferma di questo precoce interesse per le performances drammatiche, e ne consolidano il rapporto con il rituale dionisiaco. Questo tema è al centro della quarta parte (Theatron. Dionysus ex machina, 107-131), nella quale l'Autrice mostra l'evoluzione dal VI al I secolo a.C. dei materiali pertinenti, rilevando la loro profonda connessione con il rituale dionisiaco e la capillare diffusione di quest'ultimo nell'area magnogreca e siceliota. Di particolare rilievo è il periodo tra IV e III secolo quando vengono edificati, sempre all'interno dei contesti urbani, teatri in pietra che hanno la caratteristica di essere polifunzionali con una destinazione sia religiosa che politica.

Al tema della diffusione del culto in ambiente siceliota e negli altri contesti isolani, $\mathrm{S}$. Modeo dedica l'ultima ampia sezione del volume (Mysteria. La diffusione del culto dionisiaco in Sicilia tra l'età arcaica e l'età ellenistica, 133-228). Una scrupolosa rassegna delle evidenze archeologiche nelle colonie greche sottolinea la ricchezza di segni materiali rife- ribili con maggiore o minore sicurezza a presenze cultuali dionisiache. L'analisi è condotta in profondità e propone nuove chiavi di lettura, come nel caso di Monte San Mauro di Caltagirone, alla cui area appartengono tre arule fittili che riportano la scena di un personaggio maschile che toglie i piccoli ad una scrofa di cinghiale: attribuita per lo più alla sfera demetriaca, l'A. propone di cogliere un carattere dionisiaco nella vis ferina dell'uomo, che rinvia al profilo satiresco e alla religiosità dionisiaca. Dal sito di Grammichele proviene il materiale votivo rinvenuto in località Poggio dell'Aquila, che sembra pertinente ad un santuario demetriaco ma con rilevanti elementi dionisiaci. Ugualmente altre testimonianze di presenze riferibili al dio si segnalano in molte altre località, anche se non sempre è possibile inferire l'esistenza di aree cultuali organizzate, quanto piuttosto presenze collegate a santuari di altre divinità, in primis Demetra. Molto più ricca di testimonianze dionisiache si rivela il contesto siracusano, a cominciare dall'altare dedicato alla divinità in uno dei capi di Ortigia, probabilmente nella zona dell'istmo. In ogni caso è verosimile pensare che la complessiva disposizione cultuale dionisiaca in area siracusana doveva essere molto più ricca e articolata di quanto non si ricavi oggi dalle tracce materiali disponibili. Certa appare la celebrazione delle Anthesterie anche a Siracusa: lo attesta tra l'altro un frammento dello storico Timeo a proposito della celebrazione dei choes in età dionigiana (non a caso choes era il nome del secondo giorno della festa ad Atene). Per quanto concerne l'area di penetrazione siracusana, a Camarina sembra sicura la presenza del dio in associazione con Atena. In area megarese alcune indicazioni rendono verosimile il culto dionisiaco tanto a Megara che a Selinunte; e testimonianze archeologiche di pertinenza dionisiaca provengono da Monte Adranone, Rocca Nadore fino a Monte lato e Maranfusa verso il territorio imerese, a testimonianza della capacità di penetrazione selinuntina. Testimonianze di culto dionisiaco sono presenti anche a Eraclea Minoa, per la quale S. Modeo 
sottolinea l'importanza degli scavi iniziati da Salinas, che avrebbero portato alla luce un edificio teatrale (187): il santuario sulla collina sovrastante, anche se attribuito a divinità femminili, potrebbe avere una connessione con il dio del teatro. Quello che, nell'ultima parte del suo volume, l'Autrice opportunamente valorizza è la ricchezza di tracce dionisiache nei centri indigeni sottoposti all'influenza delle colonie siceliote. Un caso interessante è quello del sito di Vassallaggi, "forse l'antica Motyon distrutta da Ducezio" (206): si tratta di un centro fortemente ellenizzato nel quale il culto dionisiaco sembra ipotizzabile sulla base dei ritrovamenti archeologici provenienti dal santuario delle divinità ctonie e dai corredi tombali. Anche il sito sicano di Monte Polizzello si rivela, nell'analisi dell'A., un'area privilegiata per valutare la presenza e lo scambio con il Mediterraneo orienta in età precoloniale; risaltano in particolare i rapporti con la cultura cretese, anche in termini cultuali: le numerose protomi taurine e di ariete, insieme ad altre classi di materiali farebbero ritenere che una delle divinità minori del sito possa identificarsi con lo Zagreus di Creta, in Grecia assimilato a Dioniso. Ultimo aspetto preso in considerazione è l'altra area anellenica dell'isola, sebbene non "indigena": si tratta di quella fenicio-punica per la quale l'Autrice tiene conto opportunamente di quelle dinamiche di scambio e di interazioni culturali che, sviluppatesi tra XIII e XI sec. a.C. nel Mediterraneo orientale, costituiscono un serbatoio di stimoli e di eredità che occorre prendere in considerazione nell'analisi di questo territorio. La tradizione mitica su Cadmo e la sua stirpe sembra avere mediato il rapporto con Dioniso e culti dionisiaci appaiono attestati in diversi luoghi dell'Occidente punico, da Cartagine alla Sardegna, in connessione con le pratiche rituali nelle necropoli. II dionisismo feniciopunico troverebbe la sua migliore espressione nelle "iconografie riprodotte sulle stele portate alla luce nel santuario tophet" (213): in questo quadro Mozia si conferma un laboratorio di studio di eccezionale qualità, che conserva molte significative tracce degli scambi culturali con il mondo siceliota, in particolare selinuntino.

Chiude il lavoro la ricca bibliografia che sorregge le pagine di questo volume e l'indice dei nomi e delle località, che facilita la consultazione delle sue pagine. Ogni sezione è corredata in modo funzionale da 40 figure a colori, che confermano la buona cura editoriale.

Il volume può essere considerato una ricerca ad ampio raggio che mostra la capacità della documentazione siciliana di fornire stimoli alla ricerca in una prospettiva mediterranea: una ricerca attenta e meticolosa che Simona Modeo ha condotto con grande pazienza e con una apprezzabile sensibilità rivolta alla valorizzazione dei più piccoli documenti. L'andamento carsico con cui la documentazione dionisiaca si presenta nell'isola non impedisce perciò di chiarire la molteplice presenza dei rituali e dei culti dionisiaci e in generali la diffusione capillare del dio a tutti i livelli, da quelli sociopolitici (il teatro) fino alla sfera personale e quotidiana, nella sua valenza soterica. L'importanza di Dioniso in Sicilia era stata del resto sostenuta correttamente da Emanuele Ciaceri nel 1911: merito dell'A. è avere dato sostanza a quella giusta intuizione, forte oggi dell'enorme progresso compiuto dalla ricerca archeologica e di una rinnovata messa a punto dei dati letterari ed epigrafici. 